TRANSACTIONS OF THE

AMERICAN MATHEMATICAL SOCIETY

Volume 360, Number 6, June 2008, Pages 3133-3151

S 0002-9947(08)04459-0

Article electronically published on January 25, 2008

\title{
INVARIANTS OF CONTACT STRUCTURES FROM OPEN BOOKS
}

\author{
JOHN B. ETNYRE AND BURAK OZBAGCI
}

\begin{abstract}
In this note we define three invariants of contact structures in terms of open books supporting the contact structures. These invariants are the support genus (which is the minimal genus of a page of a supporting open book for the contact structure), the binding number (which is the minimal number of binding components of a supporting open book for the contact structure with minimal genus pages) and the norm (which is minus the maximal Euler characteristic of a page of a supporting open book).
\end{abstract}

\section{INTRODUCTION}

Emmanuel Giroux has recently found an amazing correspondence between open book decompositions of 3-manifolds and contact structures, [Gi]. Specifically, he has shown that any contact structure $\xi$ on $M$ is related to an open book through the Thurston-Winkelnkemper construction [TW]. This breakthrough has provided the basis for a much greater understanding of contact structures and 3-manifold topology.

In this note we define three new invariants of contact structures using Giroux's correspondence. Let $(M, \xi)$ be a contact manifold. The first invariant is called the support genus and is simply the minimal possible genus for a page of an open book that supports $\xi$. This invariant is denoted by $\operatorname{sg}(\xi)$. In [E1] it was shown that $\operatorname{sg}(\xi)=0$ for any overtwisted contact structure and $\operatorname{sg}(\xi)=0$ forces any symplectic filling of $(M, \xi)$ to have an intersection form that embeds in a negative definite diagonalizable quadratic form. In [OSS] it was shown that $\operatorname{sg}(\xi)=0$ implies that the Heegaard-Floer contact invariant of $\xi$ is reducible. Thus it is clear that the support genus is tied to subtle geometric properties of the contact structure. We do not add much to the understanding of support genus here.

This paper will concentrate on a second invariant, the binding number. The binding number of $\xi$ is the minimal number of binding components for an open book supporting $\xi$ and that has pages of genus $\operatorname{sg}(\xi)$. We denote the binding number by $\operatorname{bn}(\xi)$. Note that it is important to restrict the open books considered in the definition of binding number to those that have minimal genus pages, because

Received by the editors May 16, 2006.

2000 Mathematics Subject Classification. Primary 57R17.

The first author was partially supported by the NSF CAREER Grant DMS-0239600 and NSF Focused Research Grant FRG-024466.

The second author was partially supported by the Turkish Academy of Sciences and by the NSF Focused Research Grant FRG-024466. The authors thank the referee for many useful comments concerning the original version of this paper.

(c) 2008 American Mathematical Society Reverts to public domain 28 years from publication 
otherwise the binding number would always be one (any contact structure is supported by an open book with connected binding). We make several computations of the binding number.

Theorem. The binding number of an overtwisted contact structure $\xi$ on a 3manifold is bounded in terms of the Euler class of $\xi$. In particular, the infinitely many overtwisted contact structures on a manifold with Euler class zero (or torsional) take on finitely many binding numbers. Moreover, if $M$ is a rational homology sphere, then there is a universal bound, depending on $M$, on the binding number of any overtwisted contact structure on $M$.

Through examples we also show that the binding number is really an invariant of the contact structure in that it is not determined merely by the topology of the manifold $M$. We also show that in certain cases the binding number can place restrictions on the topology of the contact structure.

Theorem. If $\operatorname{sg}(\xi)=1$ and $\mathrm{bn}(\xi)=1$, then the Euler class of $\xi$ is zero.

We give an example showing that there are contact structures with $\operatorname{sg}(\xi)=1$ but having nonzero Euler class. Thus the restriction on $\mathrm{bn}(\xi)$ is necessary. This theorem follows from the following theorem.

Theorem. If $\xi$ is supported by an open book having two or fewer components in its binding and hyper-elliptic monodromy, then the Euler class of $\xi$ is zero.

The last invariant of contact structures we define is the norm. The norm of a contact structure is the negative of the maximal Euler characteristic of a page of an open book supporting $\xi$. We denote the norm by $\mathrm{n}(\xi)$. We do not know if the norm of a contact structure is determined by the support genus and binding number. We are only able to establish

$$
\min \{2 \operatorname{sg}(\xi)+\operatorname{bn}(\xi)-2,2 \operatorname{sg}(\xi)+1\} \leq \mathrm{n}(\xi) \leq 2 \operatorname{sg}(\xi)+\operatorname{bn}(\xi)-2 .
$$

Thus, when $\operatorname{bn}(\xi) \leq 3$, we know $\mathrm{n}(\xi)=2 \mathrm{sg}(\xi)+\mathrm{bn}(\xi)-2$, but in general the norm might be smaller.

In Section 2 we recall the basic definitions involving open book decompositions and Giroux's correspondence between them and contact structures. The following section discusses the definitions of the invariants of plane fields on 3-manifolds. In Section 4 we give the definition of support genus and binding number. The following two sections discuss the computation of the binding number for planar and, respectively, elliptic open books. Section 7 contains the definition of the norm of a contact structure and discusses its relation to the other two invariants. We end with several fundamental questions concerning these new invariants.

Throughout this paper we will be converting contact surgery presentations of contact structures into open book presentations of contact structures. This, in general, can be quite difficult, but all our examples can be understood using the techniques in [E1, EO].

\section{OPEN BOOK DECOMPOSITIONS}

Suppose that for an oriented link $L$ in a closed and oriented 3-manifold $M$ the complement $M \backslash L$ fibers over the circle as $\pi: M \backslash L \rightarrow S^{1}$ such that $\pi^{-1}(\theta)=\Sigma_{\theta}$ 
is the interior of a compact surface bounding $L$, for all $\theta \in S^{1}$. Then $(L, \pi)$ is called an open book decomposition (or just an open book) of $M$. For each $\theta \in S^{1}$, the surface $\Sigma_{\theta}$ is called a page, while $L$ is said to be the binding of the open book. The monodromy of the fibration $\pi$ is defined as the diffeomorphism of a fixed page, which is given by the first return map of a flow that is transverse to the pages and meridional near the binding. The isotopy class of this diffeomorphism is independent of the chosen flow, and we will refer to that as the monodromy of the open book decomposition. An open book $(L, \pi)$ on a $3-$ manifold $M$ is said to be isomorphic to an open book $\left(L^{\prime}, \pi^{\prime}\right)$ on a 3 -manifold $M^{\prime}$ if there is a diffeomorphism $f:(M, L) \rightarrow\left(M^{\prime}, L^{\prime}\right)$ such that $\pi^{\prime} \circ f=\pi$ on $M \backslash L$. In other words, an isomorphism of open books takes binding to binding and pages to pages.

An open book can also be described as follows. First consider the mapping torus

$$
\Sigma_{\phi}=[0,1] \times \Sigma /(1, x) \sim(0, \phi(x))
$$

where $\Sigma$ is a compact oriented surface with $r$ boundary components and $\phi$ is an element of the mapping class group $\Gamma_{\Sigma}$ of $\Sigma$. Since $\phi$ is the identity map on $\partial \Sigma$, the boundary $\partial \Sigma_{\phi}$ of the mapping torus $\Sigma_{\phi}$ can be canonically identified with $r$ copies of $T^{2}=S^{1} \times S^{1}$, where the first $S^{1}$ factor is identified with $[0,1] /(0 \sim 1)$ and the second one comes from a component of $\partial \Sigma$. Now we glue in $r$ copies of $D^{2} \times S^{1}$ to cap off $\Sigma_{\phi}$ so that $\partial D^{2}$ is identified with $S^{1}=[0,1] /(0 \sim 1)$, and the $S^{1}$ factor in $D^{2} \times S^{1}$ is identified with a boundary component of $\partial \Sigma$. Thus we get a closed 3-manifold $M=\Sigma_{\phi} \cup_{r} D^{2} \times S^{1}$ equipped with an open book decomposition whose binding is the union of the core circles in the $D^{2} \times S^{1}$ 's that we glue to $\Sigma_{\phi}$ to obtain $Y$. In conclusion, an element $\phi \in \Gamma_{\Sigma}$ determines a 3-manifold together with an "abstract" open book decomposition on it. Notice that by conjugating the monodromy $\phi$ of an open book on a 3 -manifold $M$ by an element in $\Gamma_{\Sigma}$ we get an isomorphic open book on a 3-manifold $M^{\prime}$ which is diffeomorphic to $M$.

Suppose that an open book decomposition with page $\Sigma$ is specified by $\phi \in \Gamma_{\Sigma}$. Attach a 1-handle to the surface $\Sigma$ connecting two points on $\partial \Sigma$ to obtain a new surface $\Sigma^{\prime}$. Let $\gamma$ be a closed curve in $\Sigma^{\prime}$ going over the new 1-handle exactly once. Define a new open book decomposition with $\phi^{\prime}=\phi \circ t_{\gamma} \in \Gamma_{\Sigma^{\prime}}$, where $t_{\gamma}$ denotes the right-handed Dehn twist along $\gamma$. The resulting open book decomposition is called a positive stabilization of the one defined by $\phi$. If we use a left-handed Dehn twist instead, then we call the result a negative stabilization. The inverse of the above process is called positive (negative) destabilization. Notice that although the resulting monodromy depends on the chosen curve $\gamma$, the 3-manifold specified by $\left(\Sigma^{\prime}, \phi^{\prime}\right)$ is diffeomorphic to the 3 -manifold specified by $(\Sigma, \phi)$.

2.1. Basic topology of a 3-manifold given as an open book. Given an abstract description of an open book in a 3-manifold $M$ with page a compact oriented genus $g$ surface $\Sigma$ with $r$ boundary components and monodromy $\phi \in \Gamma_{\Sigma}$, we can determine the basic topology of $M$ by calculating its fundamental group and its first homology group as follows. Fix a point $p_{j}$ on the $j$-th boundary component of $\partial \Sigma$, for all $j=1, \ldots, r$. We will calculate the fundamental group of $M$ based at $p_{1}$. Let $a_{1}, \ldots, a_{g}, b_{1}, \ldots, b_{g}, c_{1}, \ldots, c_{r}$ be the standard generators of $\pi_{1}(\Sigma)$ based at $p_{1}$, where the $c_{i}$ 's correspond to loops around the boundary components, and let $\theta_{j}$ denote a loop based at $p_{j}$ which is transverse to all the pages of the open book, for $j=1, \ldots, r$. Then a presentation of the fundamental group of the mapping 
torus $\Sigma_{\phi}$ can be given as $\pi_{1}\left(\Sigma_{\phi}\right)=$

$$
\left\langle a_{i}, b_{i}, c_{j}, \theta_{1} \mid \prod_{i=1}^{g}\left[a_{i} b_{i}\right] \prod_{j=1}^{r} c_{j}, \theta_{1} a_{i} \theta_{1}^{-1} \phi_{*}\left(a_{i}^{-1}\right), \theta_{1} b_{i} \theta_{1}^{-1} \phi_{*}\left(b_{i}^{-1}\right), \theta_{1} c_{j} \theta_{1}^{-1} \phi_{*}\left(c_{j}^{-1}\right)\right\rangle
$$

where $1 \leq i \leq g, 1 \leq j \leq r,\left[a_{i}, b_{i}\right]=a_{i} b_{i} a_{i}^{-1} b_{i}^{-1}$, and $\phi_{*}$ denotes the action of $\phi$ on $\pi_{1}(\Sigma)$. Now connect the base point $p_{1}$ to $p_{j}$ by an arc $\sigma_{j} \subset \Sigma$ and observe that the loop $\theta_{1} \sigma_{j} \theta_{j}^{-1} \phi_{*}\left(\sigma_{j}^{-1}\right)$ bounds a disk in $\Sigma_{\phi}$. When we cap off the boundary component of $\Sigma_{\phi}$ carrying the base point $p_{1}$ by a $D^{2} \times S^{1}$, the loop $\theta_{1}$ will clearly bound a disk in $D^{2} \times S^{1}$ and therefore it will become trivial in the resulting fundamental group. Similarly when we cap off the boundary component of $\Sigma_{\phi}$ carrying the point $p_{j}$, the above relation becomes $\sigma_{j} \phi_{*}\left(\sigma_{j}^{-1}\right)=1$. As a consequence, by capping off all the boundary components of $\Sigma_{\phi}$ by $D^{2} \times S^{1}$ 's, we get the following presentation of the fundamental group of $M$ :

$$
\pi_{1}(M)=\left\langle a_{i}, b_{i}, c_{j} \mid \prod_{i=1}^{g}\left[a_{i} b_{i}\right] \prod_{j=1}^{r} c_{j}, a_{i} \phi_{*}\left(a_{i}^{-1}\right), b_{i} \phi_{*}\left(b_{i}^{-1}\right), \sigma_{j} \phi_{*}\left(\sigma_{j}^{-1}\right)\right\rangle,
$$

where $i=1, \ldots, n$ and $j=2, \ldots, r$. Note that the relations involving the $c_{i}$ 's seems to have disappeared; this is because the monodromy map $\phi$ is the identity near the boundary. Thus these relations turn into the relations involving the $\sigma_{i}$ 's (and they show up in keeping track of base points). By abelianizing $\pi_{1}(M)$ we get a presentation of the first homology group of $M$ as:

$$
H_{1}(M)=\left\langle a_{i}, b_{i}, c_{j} \mid a_{i}-\phi_{*}\left(a_{i}\right), b_{i}-\phi_{*}\left(b_{i}\right), \sigma_{j}-\phi_{*}\left(\sigma_{j}\right)\right\rangle,
$$

where $\phi_{*}$ now denotes the action of $\phi$ on $H_{1}(\Sigma)$. Note that $c_{1}=-\left(c_{2}+\cdots+c_{r}\right)$ in $H_{1}(\Sigma)$.

2.2. Open books and contact structures. We will assume throughout this paper that a contact structure $\xi=\operatorname{ker} \alpha$ is coorientable (i.e., $\alpha$ is a global 1 -form) and positive (i.e., $\alpha \wedge d \alpha>0$ ). A contact structure $\xi$ on $M$ is said to be supported by an open book decomposition $(L, \pi)$ of $M$ if $\xi=\operatorname{ker} \alpha$ for some contact form $\alpha \in \Omega^{1}(M)$ such that $\alpha(v)>0$ for vectors inducing the positive orientation on $T L$ and $d \alpha>0$ is on every page. Thurston and Winkelnkemper [TW] have shown that every open book supports a contact structure. Recently, Giroux [Gi] has proven a converse. Specifically he has shown that every contact 3 -manifold admits a supporting open book and two open books supporting the same contact structure admit a common positive stabilization. Moreover two contact structures supported by the same open book are isotopic. We refer the reader to [E2] and [OS] for more on the correspondence between open books and contact structures.

\section{INVARIANTS OF PLANE FIELDS}

An oriented 2-plane field $\xi$ in a 3-manifold has an Euler class $e(\xi)$ (i.e., the first Chern class $\left.c_{1}(\xi)\right)$ and a 3 -dimensional invariant $d_{3}(\xi)$. When the second cohomology of the manifold has 2-torsion, the Euler class can be refined to an invariant $\Gamma(\xi)$. In this section we will describe a method to calculate these invariants for the underlying 2-plane field of a contact structure $\xi$ supported by a given open book, starting from an explicit factorization of the monodromy of the open book into Dehn twists. We begin by computing the rotation number of Legendrian knots sitting on the page of a supporting open book. 


\subsection{Rotation number of the Legendrian realization of a curve on a page.}

In this section we will compute the rotation number $r(\gamma)$ of the Legendrian realization of a homologically nontrivial curve $\gamma$ embedded on a page of an open book. We start with $P=\natural_{k} S^{1} \times D^{3}$, which is the 4 -ball $D^{4}$ union $k 1$-handles, as shown in Figure 1, where $k=2 g+r$. Evidently, $P$ is diffeomorphic to $D^{2} \times \Sigma$, where $\Sigma$ is a compact oriented surface of genus $g$ with $r$ boundary components. Note that $\partial P=\partial\left(D^{2} \times \Sigma\right)=\partial D^{2} \times \Sigma \cup D^{2} \times \partial \Sigma$ is a canonical decomposition of $\partial P$ into an open book. The monodromy of this open book on $\partial P$ is the identity map.

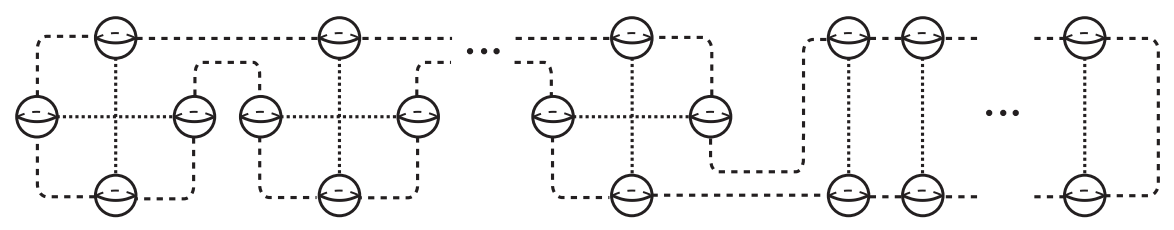

Figure 1. 4-ball $D^{4}$ union $k 1$-handles. The dashed line indicates the boundary of $\Sigma$ and dotted lines indicate generators of the homology of $\Sigma$.

The way the attaching balls of the 1 -handles are identified gives a trivialization of the tangent bundle of $P$. This trivialization also induces a trivialization of the tangent bundle of the page which is depicted in Figure 1 with its orientation induced from the blackboard: just take the usual oriented frame of $\mathbb{R}^{2}$, restrict it to the disk and extend over the 1-handles.

Next we apply the construction of Thurston and Winkelnkemper in [TW] to put a contact structure $\xi$ on $\partial P=\#_{k} S^{1} \times S^{2}$ supported by its canonical trivial open book decomposition: Away from the binding the contact 1 -form is given by $\alpha=\beta+d \theta$, where $\theta$ is the meridional direction to the binding, and $\beta$ is a 1 -form transverse to the binding such that $d \beta$ is an area form on $\Sigma$. The pages of the open book are convex and $\partial_{\theta}$ is the Reeb vector field which is transverse to the pages.

Since the contact structure $\xi$ is supported by the trivial open book on $\partial P$ by construction, we can assume that the tangents to the pages are as close as we wish to the contact planes, away from the binding (cf. [E2]). This gives a trivialization of the contact planes restricted to a page. Recall that the rotation number of a Legendrian curve is the winding number of its tangent vector with respect to a fixed trivialization of the contact structure over the curve. (Note the contact planes can be globally trivialized since $e(\xi)=0$.) On the other hand a smooth curve on a surface has a winding number which is defined as the winding number of its tangent vector with respect to a fixed trivialization of the tangent bundle of the surface. We conclude that the rotation number of a Legendrian knot $\gamma$ is the same as the winding number of the projection of $\gamma$ to a page $\Sigma$ since we can use the same trivialization to define both invariants. This winding number can be calculated for any curve $\gamma$ on the page, since we fix a trivialization of the tangent bundle of the page once we fix the attaching regions of the 1-handles in Figure 1.

3.2. Calculation of Euler class from monodromy. Consider an open book with page a genus $g$ surface $\Sigma$ with $r$ boundary components and monodromy $\phi$. It is well known that we can factor $\phi$ as a product of Dehn twists along homologically 
nontrivial curves $\gamma_{1}, \gamma_{2}, \cdots, \gamma_{n}$ on $\Sigma$. Below we will describe a method to calculate the Euler class $e(\xi)$ (i.e., $c_{1}(\xi)$ ) of the underlying 2-plane field of the contact structure $\xi$ supported by this open book.

Note that an open book is the boundary of an achiral Lefschetz fibration whose handlebody description as a 4-manifold is discussed in detail in [GS]. The curves $\gamma_{1}, \gamma_{2}, \cdots, \gamma_{n}$ which appear in the factorization of the monodromy $\phi$ of the given open book are the vanishing cycles of this achiral Lefschetz fibration. Consider the $\gamma_{i}$ 's as embedded in distinct pages of the trivial open book on $\partial P=\#_{k} S^{1} \times S^{2}$, where $P=\natural_{k} S^{1} \times D^{3}$, as depicted in Figure 1. We Legendrian realize each of these curves on distinct pages. Then applying contact $( \pm 1)$-surgery on $\gamma_{i}$ corresponds to adding a vanishing cycle to the achiral Lefschetz fibration bounding the open book. If we apply (-1)-surgeries only we get an "honest" Lefschetz fibration $X$ and the almost complex structure on $P$ will extend to $X$. The Euler class of the contact structure $\xi$ is the restriction of $c_{1}(X, J)$ to $\partial X=M$, and its Poincaré dual can be given by

$$
P D(e(\xi))=\sum_{i=1}^{n} r\left(\gamma_{i}\right)\left[\mu_{i}\right] \in H_{1}(M, \mathbb{Z})
$$

where $\mu_{i}$ is the meridian of $\gamma_{i}$.

If there exist $(+1)$-surgeries, then although we cannot find an almost complex structure on the achiral Lefschetz fibration $X$, there is an almost complex structure on $X \# q \mathbb{C} P^{2}$ (cf. $[\mathrm{OS}]$ ), where $q$ is the number of $(+1)$-surgeries. The Poincaré dual $P D(e(\xi))$ will be evaluated in the same way as above by restricting the first Chern class of the almost complex structure on $X \# q \mathbb{C} P^{2}$ to the boundary.

When the second cohomology of $M$ has two torsion, then $e(\xi)$ is not the complete two dimensional invariant of $\xi$. In $[\mathrm{G}]$ a refinement of $e(\xi)$ was given. This invariant is a map $\Gamma(\xi)$ from the spin structures on $M$ to $G=\left\{c \in H^{2}(M ; Z) \mid 2 c=e(\xi)\right\}$. If $X$ is a Stein 2-handlebody obtained by attaching 2-handles to $D^{4}$ along a Legendrian link $L=\left\{K_{1}, \ldots, K_{n}\right\}$ in $S^{3}=\partial D^{4}$, then $\Gamma$ can be described as follows: A spin structure $\mathfrak{s}$ on $M=\partial X$ is described by a characteristic sub-link $L_{\mathfrak{s}} \subset L$; see [GS]. Then $\Gamma(\xi)(\mathfrak{s})$ is the restriction to $M$ of the class $\rho \in H^{2}(X ; Z)$ determined by

$$
\left\langle\rho, \alpha_{i}\right\rangle=\frac{1}{2}\left(r\left(K_{i}\right)+\operatorname{lk}\left(K_{i}, L_{\mathfrak{s}}\right)\right),
$$

where $\alpha_{i}$ is the homology class in $X$ determined by $K_{i}$.

3.3. The 3-dimensional invariant. The 3 -dimensional invariant $d_{3}(\xi)$ of a plane field is a rational number well defined modulo the divisibility of $e(\xi)$. We will only describe how to compute $d_{3}(\xi)$ when $e(\xi)$ is a torsion element and thus $d_{3}(\xi)$ is well defined. Let $M$ and $X$ be as above. Then we have

$$
d_{3}(\xi)=\frac{1}{4}\left(c^{2}(X)-3 \sigma(X)-2 \chi(X)\right)+q,
$$

where $\sigma$ is the signature of $X, \chi$ is the Euler characteristic, and $q$ is the number of $(+1)$-surgeries. The number $c^{2}(X)$ is the square of the class $c(X)$ with Poincaré dual

$$
\sum_{i=1}^{k} r\left(\gamma_{i}\right) C_{i}
$$

where the $C_{i}$ 's are the cocores of the 2-handles attached along the $\gamma_{i}$ 's. Note that $\left.c(X)\right|_{M}=e(\xi)$, which we are assuming to be a torsion class. Thus some multiple 
$k c(X)$ of $c(X)$, which naturally lives in $H^{2}(X ; \mathbb{Z})$, comes from a class $c_{r}(X)$ in $H^{2}(X, \partial X ; \mathbb{Z})$ which can be squared. So $c^{2}(X)$ means $\frac{1}{k^{2}} c_{r}^{2}(X)$. Formula $(3.2)$ is a slight generalization of the one given in [DGS], where it was assumed that $X$ had no 1-handles. Their proof carries over to our case; see $[\mathrm{EF}]$.

\section{INVARIANTS OF CONTACT STRUCTURES FROM OPEN BOOKS}

There are several obvious invariants one can define using open book decompositions associated to contact structures. We begin with the support genus.

Definition 4.1. The support genus of a contact structure $\xi$ on a 3 -manifold $M$ is the minimal genus of a page of an open book decomposition of $M$ supporting $\xi$,

$$
\operatorname{sg}(\xi)=\min \{\operatorname{genus}(\Sigma) \mid(\Sigma, \phi) \text { an open book decomposition supporting } \xi\} .
$$

This definition was implicitly given and studied in [E1] where the following was shown.

Theorem 4.2. If $(M, \xi)$ is a fillable contact structure and $\operatorname{sg}(\xi)=0$, then any filling of $(M, \xi)$ (1) has only one boundary component, (2) has negative definite intersection form and (3) the intersection form embeds in a diagonalizable form.

Theorem 4.3. If $(M, \xi)$ is overtwisted, then $\operatorname{sg}(\xi)=0$.

There are many examples of contact structures on lens spaces and Seifert fibered spaces that have genus zero $[\mathrm{E} 1, \mathrm{~S}]$. There are also many examples of contact structures having genus one [E1, EO]. Surprisingly, though there are many potential examples [EO], it is still unknown if $\operatorname{sg}(\xi)>1$ for any contact structure $\xi$.

Next we define the binding number of a contact structure.

Definition 4.4. The binding number of a contact structure $\xi$ on a 3-manifold $M$ is the minimal number of components in the binding of the open book decomposition supporting $\xi$ that have minimal genus,

$$
\begin{gathered}
\operatorname{bn}(\xi)=\min \{|\partial \Sigma| \mid(\Sigma, \phi) \text { an open book decomposition supporting } \\
\xi \text { with genus }(\Sigma)=\operatorname{sg}(\xi)\} .
\end{gathered}
$$

To illustrate the difficulty in computing the binding number of a contact structure we give a simple example. Consider the contact structures $\xi_{1}$ and $\xi_{2}$ in $S^{3}$ described by their contact surgery diagrams depicted in Figure 3. The contact structure $\xi_{1}$ can be given equivalently by two contact $(+1)$-surgeries performed on the same unknot with its Legendrian push-off. By computing the 3-dimensional invariants of $\xi_{i}$ we can see that $\xi_{1}$ is isotopic to $\xi_{2}$ and both are overtwisted.
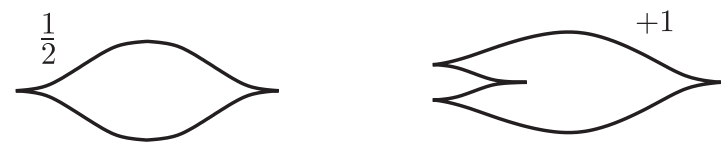

Figure 2. Two isotopic overtwisted contact structures $\xi_{1}$ (on the left) and $\xi_{2}$ (on the right) in $S^{3}$.

The open books $\mathfrak{o b}_{1}, \mathfrak{o b}_{2}$ supporting $\xi_{1}, \xi_{2}$ are given in Figure 3, on the upper left and lower left, respectively. (To see that these open books are associated to the surgery presentations in Figure 2, see [EO].) The \pm sign on a curve indicates 
a Dehn twist along that curve - right-handed for plus and left-handed for minus sign. Thus it appears that $\operatorname{bn}\left(\xi_{2}\right)=3$, but in fact $\operatorname{bn}\left(\xi_{2}\right)=2$. Using Giroux's correspondence we know that $\mathfrak{o b}_{1}$ and $\mathfrak{o b}_{2}$ are equivalent up to stabilization. This is easily seen. If we positively stabilize $\mathfrak{o b}_{1}$ twice and $\mathfrak{o b}_{2}$ once, then we can see that the resulting open books are isomorphic by the lantern relation on the four-holed sphere as illustrated in Figure 3.
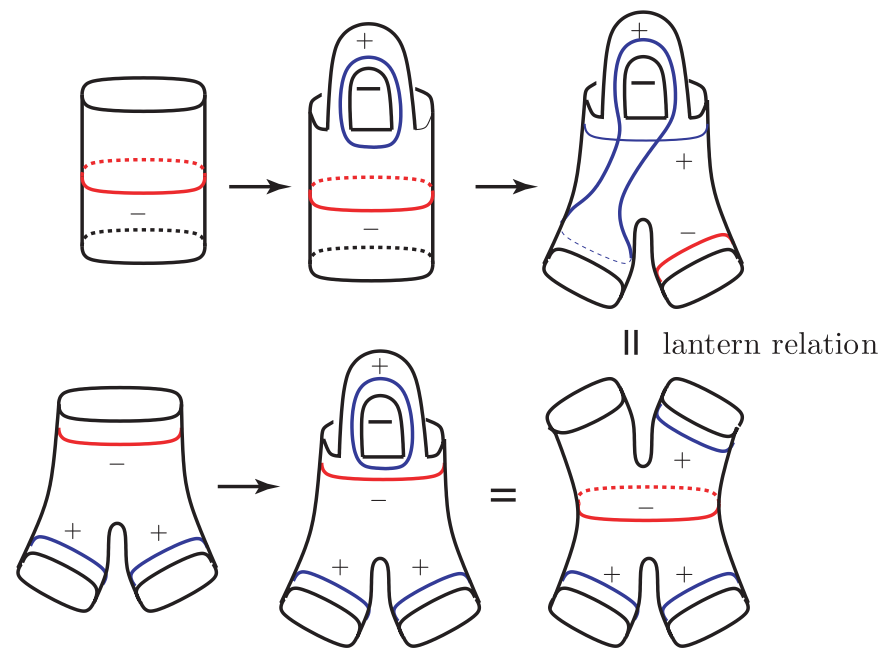

Figure 3. Positive stabilization of $\mathfrak{o b}_{1}$ twice (the top row) and $\mathfrak{o b}_{2}$ once (the bottom row).

\section{Planar open Books}

We first consider the binding number for contact structures supported by planar open books. Our main goal in this section is to give a bound on the binding number for overtwisted contact structures and see that the binding number gives a nontrivial invariant of a contact structure, that is, that the binding number of a contact structure can be larger than that which is forced by the topology of the manifold. We begin with a simple lemma.

Lemma 5.1. Suppose $\xi$ is a contact structure on a 3-manifold $M$ that is supported by a planar open book.

(1) If $\mathrm{bn}(\xi)=1$, then $\xi$ is the standard tight contact structure on $S^{3}$.

(2) If $\operatorname{bn}(\xi)=2$ and $\xi$ is tight, then $\xi$ is the unique tight contact structure on $L(p, p-1)$ for some $p$.

(3) If $\mathrm{bn}(\xi)=2$ and $\xi$ is overtwisted, then $\xi$ is the overtwisted contact structure on $L(p, 1)$, for some $p$, with $e(\xi)=0$ and $d_{3}(\xi)=-\frac{1}{4} p+\frac{3}{4}$. When $p$ is even, then the refinement of $e(\xi)$ is given by $\Gamma(\xi)(\mathfrak{s})=\frac{p}{2}$ where $\mathfrak{s}$ is the unique spin structure on $L(p, 1)$ that extends over a two handle attached to $\mu$ with framing zero. Here we are thinking of $L(p, 1)$ as $-p$ surgery on an unknot and $\mu$ is the meridian to the unknot.

Proof. Statement (1) is obvious. An open book with annular pages supports a tight contact structure if and only if the monodromy is $t_{c}^{p}$ for some $p \geq 0$, where $t_{c}$ is 
a right handed Dehn twist about the core circle $c$ in the annulus. The contact structure supported by such an open book can also be obtained by Legendrian surgery on $p-1$ copies of the Legendrian unknot with $t b=-1$. This manifold is easily seen to be $L(p, p-1)$, and the contact structure must be the unique tight contact structure on it.

Similarly for $p>0$, the contact structure supported by an open book with monodromy $t_{c}^{-p}$ can also be obtained by $(+1)$-contact surgery on $p+1$ copies of the Legendrian unknot with $t b=-1$. Thus the manifold will be $L(p, 1)$, and the contact structure will be overtwisted. The invariants $\Gamma$ and $d_{3}$ are easily computed from Equations (3.1) and (3.2).

Theorem 5.2. Let $\xi$ be the contact structure on the lens space $L(4,1)$ obtained from contact surgery on the Legendrian unknot with $t b=-3$ and $r=0$. Then $\operatorname{sg}(\xi)=0$ and $\operatorname{bn}(\xi)=4$. However there is an open book for $L(4,1)$ that has annular pages.

This theorem indicates that the binding number of a contact structure is sensitive to the plane field $\xi$ and is not determined by the topology of the manifold. However, it turns out that an open book for $L(4,1)$ that supports a plane field homotopic to $\xi$ with fewer than four boundary components does not exist. So bn $(\xi)$ is still determined by the topology of the plane field. It is interesting to note that there is an overtwisted contact structure $\xi^{\prime}$ on $L(4,1)$ with bn $\left(\xi^{\prime}\right)=2$ with $e(\xi)=e\left(\xi^{\prime}\right)$ and $d_{3}(\xi)=d_{3}\left(\xi^{\prime}\right)$. However, $\Gamma(\xi) \neq \Gamma\left(\xi^{\prime}\right)$.

Proof. One may easily construct an open book decomposition for $\xi$. See Figure 4. From this open book we see that $\operatorname{bn}(\xi) \leq 4$. From Lemma 5.1 we see that $b n(\xi)>2$.
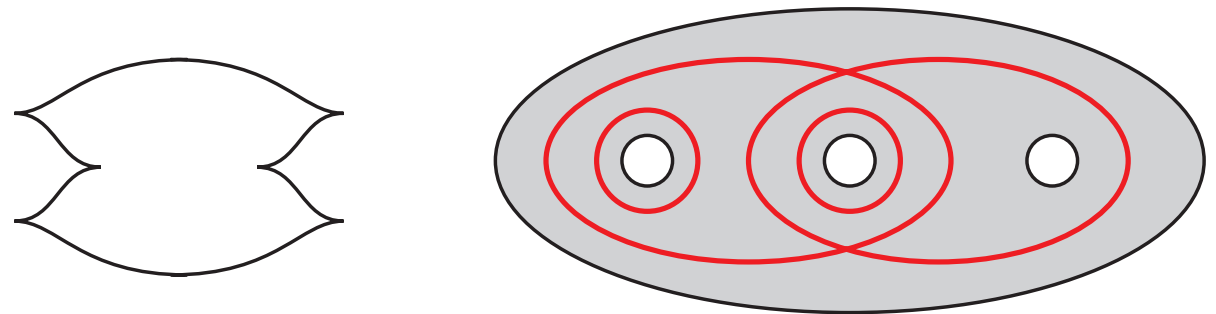

FIGURE 4. The contact structure $\xi$ on $L(4,1)$ is obtained by Legendrian surgery on the knot shown on the left. On the right is an open book supporting $\xi$. The monodromy is a right handed Dehn twist about each curve shown.

Thus we must rule out the possibility $\mathrm{bn}(\xi)=3$. To this end the surgery picture for manifolds given by open books with planar page having three boundary components is given in Figure 5. For the supported contact structure to be tight we must have $m, n$ and $k$ all be nonnegative (otherwise, the open book will be non-right veering $[\mathrm{HKM}])$. For this manifold to have a first homology group of order 4 , we must have $(m, n, k)=(0,4,1)$ or $(0,2,2)$. (Note the surgery picture is symmetric in $m, n$ and $k$, so we do not list all permutations.) One may easily check that $(m, n, k)=(0,4,1)$ yields $L(4,3)$ and $(m, n, k)=(0,2,2)$ yields $L(2,1) \# L(2,1)$, so $\xi$ cannot be supported by a planar open book with three boundary components.

Recall that the set of homotopy classes of plane fields on a 3-manifold $M$ with a fixed Euler class $e$ (or fixed refinement $\Gamma$ if there is 2-torsion in $H^{2}(M ; \mathbb{Z})$ ) is 
in one-to-one correspondence with $\mathbb{Z}_{d}$ where $d$ is the divisibility of $e$ in $H^{2}(M ; \mathbb{Z})$. (Here $\mathbb{Z}_{d}$ will mean $\mathbb{Z}$ when $e$ is a torsion element in $H^{2}(M ; \mathbb{Z})$.) Thus every manifold has infinitely many homotopy classes of plane fields, and hence infinitely many overtwisted contact structures. From [E1] we know that the support genus of any overtwisted contact structure is always zero. It seems reasonable to believe that the binding number of an overtwisted contact structure should be related to the homotopy class of the underlying plane field. The next theorem indicates this relation is very weak.

Theorem 5.3. The binding number of an overtwisted contact structure $\xi$ on a 3-manifold is bounded in terms of the Euler class of $\xi$. In particular, the infinitely many overtwisted contact structures on a manifold with Euler class zero (or torsional) take on finitely many binding numbers.

Corollary 5.4. If $M$ is a rational homology sphere, then there is a universal bound, depending on $M$, on the binding number of any overtwisted contact structure on $M$.

To prove this theorem we first study the overtwisted contact structures on $S^{3}$. The range of $d_{3}$ for plane fields on $S^{3}$ is $\mathbb{Z}+\frac{1}{2}$, with the homotopy class containing the standard tight contact structure having $d_{3}=-\frac{1}{2}$. We denote the unique overtwisted contact structure on $S^{3}$ with three dimensional invariant $d_{3}$ by $\xi_{d_{3}}$.

Lemma 5.5. For any overtwisted contact structure $\xi$ on $S^{3}$ we have

$$
2 \leq \operatorname{bn}(\xi) \leq 6 .
$$

More precisely, we have

$$
\begin{aligned}
\operatorname{bn}\left(\xi_{\frac{1}{2}}\right) & =2, \\
\operatorname{bn}\left(\xi_{-\frac{1}{2}}\right)=\operatorname{bn}\left(\xi_{\frac{3}{2}}\right) & =3, \\
\operatorname{bn}\left(\xi_{\frac{5}{2}}\right) & =4,
\end{aligned}
$$

and

$$
\begin{array}{ll}
4 \leq \operatorname{bn}\left(\xi_{\frac{4 p+1}{2}}\right) \leq 5, & p \neq 0, \\
4 \leq \operatorname{bn}\left(\xi_{\frac{4 p+3}{2}}\right) \leq 6, & p \neq-1,0 .
\end{array}
$$

Note that this lemma says Theorem 5.3 is true for $S^{3}$.

Proof. From Lemma 5.1 we have $\mathrm{bn}\left(\xi_{\frac{1}{2}}\right)=2$ and bn $>2$ for all other overtwisted contact structures on $S^{3}$. We next analyze the planar open books for $S^{3}$ with three binding components. Let $\Sigma$ be the planar surface with three boundary components. Any diffeomorphism of $\Sigma$ is determined by three numbers $m, n, k$ that give the number of Dehn twists on curves $\gamma_{1}, \gamma_{2}, \gamma_{3}$ parallel to each boundary component. Let $M_{m, n, k}$ be the 3-manifold determined by the open book with page $\Sigma$ and monodromy given by $t_{\gamma_{1}}^{m} t_{\gamma_{2}}^{n} t_{\gamma_{3}}^{k}$. It is easy to see that $M_{m, n, k}$ is the Seifert fibered space shown in Figure 5. If $M_{m, n, k}$ is diffeomorphic to $S^{3}$, then we claim $|m|,|n|,|k|$ cannot all be larger than one. Indeed, assume this is the case and further assume that $m$ and $n$ are positive (this argument is clearly symmetric in $m, n$ and $k$ so no generality is lost). Figure 6 gives a handle decomposition of a 4 -manifold $X$ with boundary $M_{m, n, k}$. Moreover, if $k$ is negative, then this decomposition can be realized by Legendrian knots with Thurston-Bennequin invariants one larger than the framings. Thus $X$ has a Stein structure. Since the only Stein filling of $S^{3}$ has $b_{2}=0$ (see [E]) the manifold $M_{m, n, k}$ cannot be $S^{3}$. If $k$ is not negative, then we 

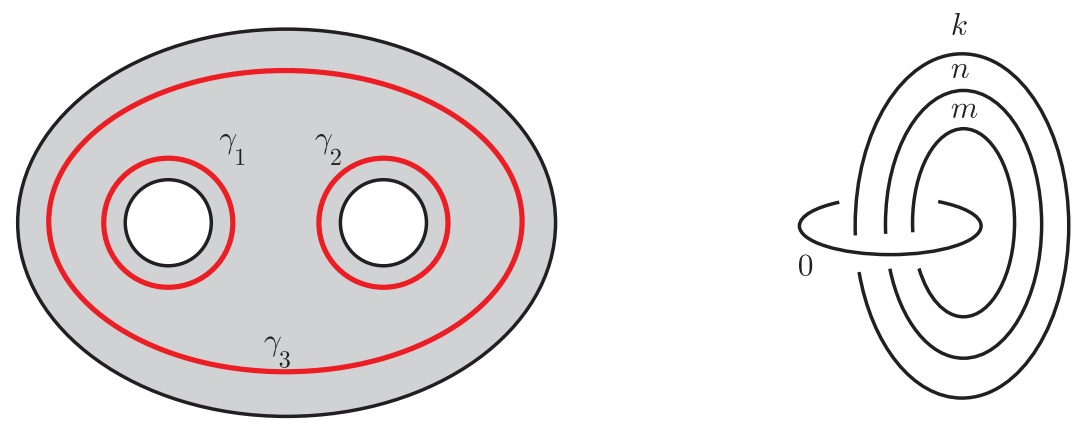

Figure 5. The surface $\Sigma$, left. The manifold $M_{m, n, k}$, right.

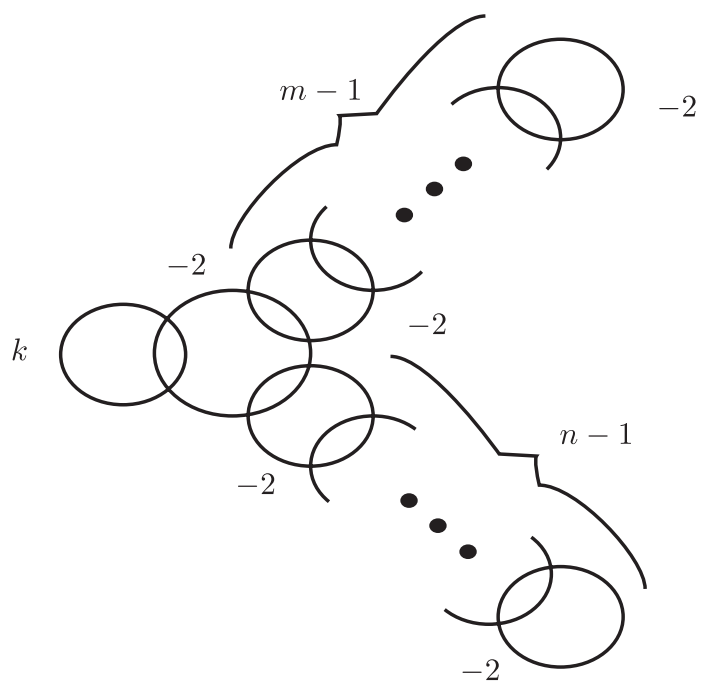

Figure 6. Handle decomposition of a 4-manifold $X$ with $\partial X=M_{m, n, k}$.

may expand the $k$-framed circle into a leg of $k-1,-2$-framed unknots (as we did for the $m$ and $n$ framed unknots to go from Figure 5 to Figure 6 ) changing the framing on the central unknot to -3 . Finally, if $m$ and $n$ are less than -2 , we can reverse the orientation on $M_{m, n, k}$ and argue as above.

To determine the contact structures realized from our open book for $M_{m, n, k}$ we note that the corresponding contact surgery picture is given in Figure 7. (Recall $\frac{1}{m}$ surgery on a Legendrian knot is performed by taking $m$ Legendrian push-offs of the Legendrian knot and performing $(+1)$, respectively $(-1)$, contact surgery if $m$ is positive, respectively negative.) We now know that $m, n$ or $k$ must be $0, \pm 1$. If one of these is zero, then the other two must be \pm 1 . In this case we realize the invariants $\frac{1}{2}($ with $(m, n, k)=(-1,1,0)), \frac{3}{2}$ (with $\left.(m, n, k)=(-1,-1,0)\right)$ and $-\frac{1}{2}$ (with $(m, n, k)=(0,1,1))$. However, the contact structure with $d_{3}=-\frac{1}{2}$ is tight.

The other possibilities for $(m, n, k)$ are $(1, j,-1),(1,-1, j),(-1, j, 1)$, where $j$ is any integer, and $(3,2,-1),(3,-1,2),(2,-1,3),(-2,-3,1),(-2,1,-3)$ and $(-3,1,-2)$. (Note interchanging $m$ and $n$ does not affect the contact structure in Figure 7; thus we do not list possibilities for $(m, n, k)$ that differ by switching $m$ 


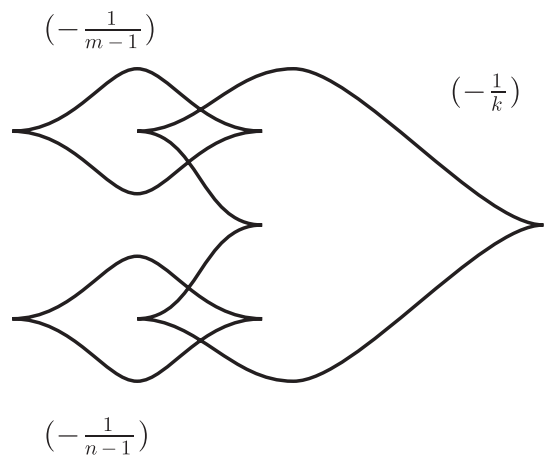

FigURE 7. A contact surgery picture of $M_{m, n, k}$.

and $n$. Actually, any permutation of the triple $(m, n, k)$ will lead to contactomorphic contact structures, so one only really needs to check three cases.) For the first three sets of possibilities we get an overtwisted contact structure with $d_{3}=\frac{1}{2}$, for the next three we get an overtwisted contact structure with $d_{3}=\frac{3}{2}$, and for the last three we get an overtwisted contact structures with $d_{3}=-\frac{1}{2}$.

Now for any overtwisted contact structure on $S^{3}$ with $d_{3} \neq-\frac{1}{2}, \frac{1}{2}$ or $\frac{3}{2}$ we know that $b n \geq 4$. By negatively stabilizing the open book for $\xi_{\frac{3}{2}}$ with three boundary components we see that $b n\left(\xi_{\frac{5}{2}}\right)=4$. For the other overtwisted contact structures let $\xi_{p}$ be the contact structure shown in Figure 8. It is easy to check that $\xi_{p}$ is a contact structure on $S^{3}$. Let $\Sigma$ be the surface shown on the right of Figure 8 and
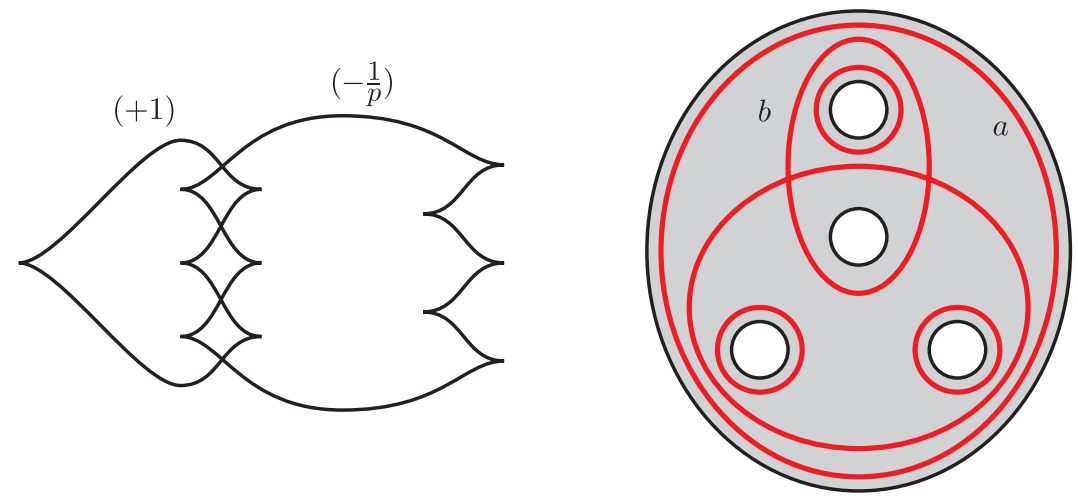

Figure 8. A contact surgery picture of $\xi_{p}$ on the left. On the right is a surface $\Sigma$.

$\phi=t_{b}^{p} t_{a}^{-1} \psi$, where $a$ and $b$ are the marked curves and $\psi$ is the diffeomorphism of $\Sigma$ consisting of a right-handed Dehn twist about the four unmarked curves. The open book decomposition $(\Sigma, \phi)$ supports the contact structure obtained by the contact surgery diagram on the left in Figure 8 (see [E1, EO]). From Figure 8 one may easily compute

$$
d_{3}\left(\xi_{p}\right)=\frac{-4 p+1}{2}
$$


Thus $\operatorname{bn}\left(\xi_{\frac{-4 p+1}{2}}\right) \leq 5$. If we negatively stabilize $(\Sigma, \phi)$ we get an open book with planar pages for $\xi_{\frac{-4 p+3}{2}}$ showing that $\operatorname{bn}\left(\xi_{\frac{-4 p+3}{2}}\right) \leq 6$.

Remark 5.6. When $(m, n, k)=(1,-1, j)$ in Figure 7 , one obtains the same overtwisted contact structure on $S^{3}$ independent of $j \in \mathbb{Z}$. Thus we have an infinite family of open books $\mathfrak{o} \mathfrak{b}_{j}$ for a single contact structure. According to Giroux any two in this family will become isotopic after some number of positive stabilizations. Using the lantern relation as we did in Figure 3 one can see that $j-j^{\prime}$ positive stabilizations will be sufficient to make $\mathfrak{o b}_{j}$ and $\mathfrak{o b}_{j^{\prime}}$ isotopic.

On the other hand, the open books $\mathfrak{o b}_{p}$ in Figure 8 give an infinite family of distinct contact structures with different $d_{3}$ invariants. Thus it is clear that $\mathfrak{o b}_{p}$ must be negatively stabilized $p^{\prime}-p$ times to even give a contact structure homotopic to the one supported by $\mathfrak{o b}_{p^{\prime}}$ where $p^{\prime}>p$. Thus for the open books $\mathfrak{o b}_{p}$ and $\mathfrak{o} \mathfrak{b}_{p^{\prime}}$ to give isotopic open books, $\mathfrak{o b}_{p}$ must be stabilized at least $p^{\prime}-p$ times. As a consequence we get simple examples of fibered knots needing arbitrarily many "Hopf plumbings" before they become isotopic. These examples are simpler than those produced in $[\mathrm{NR}]$.

Proof of Theorem 5.3. Given two contact structures $\xi_{1}, \xi_{2}$ on $M^{3}$ that are homotopic, as plane fields, over the two skeleton of $M$ there is an overtwisted contact structure $\xi^{\prime}$ on $S^{3}$ such that $\xi_{1} \# \xi^{\prime}$ is homotopic to $\xi_{2}$ as plane fields on all of $M$. Thus given any overtwisted contact structure $\xi$ on $M$ we know that it is supported by a planar open book with, say, $k$ boundary components. By Murasugi summing the open book from Lemma 5.5 we get open book decompositions for all overtwisted contact structures on $M$ with Euler class $e=e(\xi)$ having less than $k+7$ components in its binding. Hence we have a bound for bn .

\section{ELLIPTIC AND HYPER-ELLIPTIC OPEN BOOKS}

We will call an open book whose page is genus one an elliptic open book. An open book whose monodromy commutes with the hyper-elliptic involution will be called hyper-elliptic. In this section we consider restrictions on the Euler class of a contact structure that is supported by an elliptic or hyper-elliptic open book.

6.1. Elliptic open books. From our discussion of rotation numbers in Section 3.1 we have the following simple observations.

Lemma 6.1. If $(M, \xi)$ is supported by an elliptic open book with connected binding, then $e(\xi)=0$.

Proof. The monodromy $\phi$ of an elliptic open book with connected binding can be expressed as a product of Dehn twists along the curves $a$ and $b$ depicted in Figure 9. But since the winding numbers of $a$ and $b$ on the page are clearly both zero, the rotation numbers of their Legendrian realizations are also zero, and thus $e(\xi)=0$.

It was conjectured that the Euler class of a fillable contact structure supported by an elliptic open book is torsion. While the lemma above verifies this conjecture if $b n=1$, it is not always true when the binding number is larger than one, as the following example shows. 


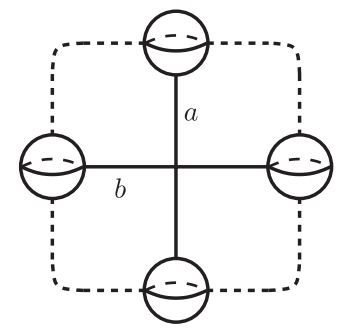

Figure 9. Page of an elliptic open book with connected binding.

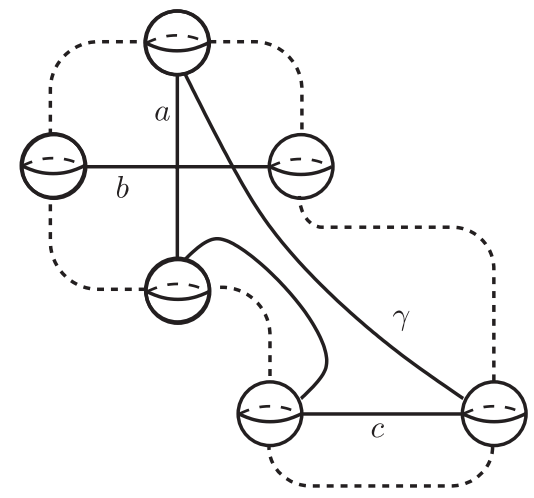

Figure 10. Page of an elliptic open book with two binding components.

Let $\Sigma_{1,2}$ denote a genus one surface with two boundary components as in Figure 10 and let

$$
\phi=\left(t_{a} t_{b}\right)^{5} t_{\gamma}^{2} t_{c}^{2}: \Sigma_{1,2} \rightarrow \Sigma_{1,2} .
$$

Let $(M, \xi)$ be the contact 3-manifold given by the open book specified by $\left(\Sigma_{1,2}, \phi\right)$.

Theorem 6.2. The support genus and binding number of $(M, \xi)$ are

$$
\operatorname{sg}(\xi)=1, \quad \operatorname{bn}(\xi)=2 .
$$

Proof. We begin by computing the homology of $M$ using the presentation in Section 2.1. The generators of $H_{1}(M)$ are $a, b$ and $c$ and the relations are

$$
a+b=\phi_{*}(a)=a,-a+2 b+2 \gamma=\phi_{*}(b)=b, 2 c+2 \gamma=\phi_{*}(\sigma)-\sigma=0,
$$

where $\sigma$ is a curve connecting the two boundary components. Hence we get

$$
H_{1}(M)=\langle a, c \mid a=-2 c\rangle=\langle\gamma\rangle=\mathbb{Z},
$$

where $\gamma=a+c$. On the other hand, when we apply the algorithm in Section 3.2, the Euler class $e(\xi)$ of the supported Stein fillable contact structure $\xi$ is given by $2 \gamma$ since the rotation numbers (i.e., the winding numbers) of $a, b$ and $c$ are zero while the rotation number of $\gamma$ is equal to one. Clearly $e(\xi)=2 \gamma$ is nontorsion in $H_{1}(M)$. Note that $(M, \xi)$ cannot be supported by an elliptic open book with connected binding by Lemma 6.1 .

We claim that $(M, \xi)$ is not supported by a planar open book either. Consider the elliptic surface $E(1)$ with nine disjoint sections. The monodromy of the elliptic fibration $E(1) \rightarrow S^{2}$ can be given by $\left(t_{b} t_{a}\right)^{6}$, where $a$ and $b$ denote the standard 
generators of the first homology group of a fiber. By removing the union of a section and a cusp fiber from $E(1)$ we get an elliptic fibration on the 4 -manifold $W$ with once punctured torus fibers whose monodromy is $\left(t_{b} t_{a}\right)^{5}$. One can check that $\partial W$ is diffeomorphic to $\Sigma(2,3,5)$ by Kirby calculus (see, for example, [GS]). Thus there is an induced open book on $\Sigma(2,3,5)$ with monodromy $\left(t_{b} t_{a}\right)^{5}$. Since the monodromy of this open book is a product of right-handed Dehn twists only, the contact structure supported by this open book is Stein fillable (cf. [Gi]) and hence isotopic to the unique tight contact structure $\xi^{\prime}$ on $\Sigma(2,3,5)$. Note that, by [E1], $\xi^{\prime}$ cannot be supported by a planar open book. By positively stabilizing the open book with monodromy $\left(t_{b} t_{a}\right)^{5}$ we get an open book (still supporting $\xi^{\prime}$ ) with two binding components whose page is $\Sigma_{1,2}$ and whose monodromy is given by $\left(t_{a} t_{b}\right)^{5} t_{c}$. We now observe that $(M, \xi)$ is obtained from $\left(\Sigma(2,3,5), \xi^{\prime}\right)$ by Legendrian surgeries. Hence (the proof of) Theorem 1.2 of [E1] implies that $(Y, \xi)$ is not supported by a planar open book either. This fact also follows from Corollary 1.4 in [OSS], since $e(\xi)$ is nontorsion and $c^{+}(\xi) \neq 0$ (because $\xi$ is Stein fillable).

6.2. Hyper-elliptic open books. Recall that the hyper-elliptic involutions $h_{1}$ : $\Sigma_{g, 1} \rightarrow \Sigma_{g, 1}$ and $h_{2}: \Sigma_{g, 2} \rightarrow \Sigma_{g, 2}$ on a surface with one or two boundary components, respectively, are the involutions shown in Figure 11. Note if the surface has
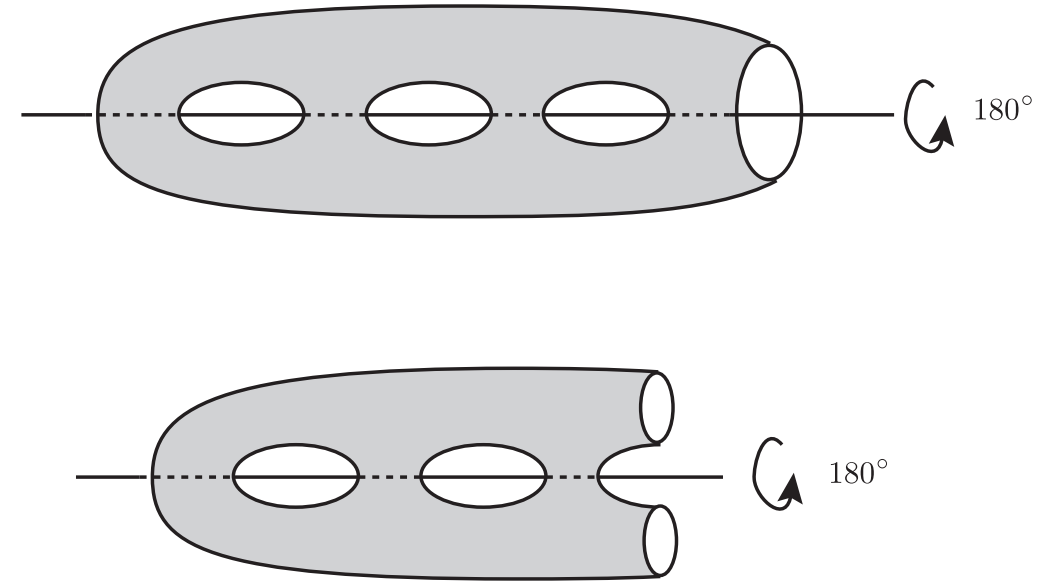

Figure 11. The hyper-elliptic involution $h_{1}$ top, and $h_{2}$ bottom.

genus $g$, then $h_{1}$ is the covering translation for the unique 2-fold branched cover over $D^{2}$ with $2 g+1$ branch points and $h_{2}$ is the covering translation for the unique 2 -fold branch cover of $D^{2}$ with $2 g$ branch points. We call an open book $\left(\Sigma_{g, i}, \phi\right)$ hyper-elliptic if $\phi \circ h_{i}=h_{i} \circ \phi$, for $i=1,2$. Lemma 6.1 above is a special case of the following theorem.

Theorem 6.3. Let $(M, \xi)$ be supported by a hyper-elliptic open book. Then $e(\xi)=$ 0 .

Remark 6.4. We note the converse of this theorem is not true. Let $\xi$ be the Stein fillable contact structure on $T^{3}$. It is well known that $e(\xi)=0$ but $\xi$ cannot be supported by a hyper-elliptic open book since that would imply $T^{3}$ is a 2 -fold branch cover over $S^{3}$ (see the lemma below); but $T^{3}$ is not a 2 -fold (or any cyclic) branch cover over $S^{3}$, [HN]. 
Before proving the theorem we recall the definition of a contact branched cover, $[\mathrm{Ge}$. Let $(M, \xi)$ be a contact 3 -manifold and $\Gamma$ be a transverse link in it. Let $p: M^{\prime} \rightarrow M$ be a covering map branched over $\Gamma$. There is a standard neighborhood $N$ of $\Gamma$ and $M^{\prime} \backslash p^{-1}(N)$ is a covering space of $M \backslash N$. Thus we may pull $\xi$ back to $M^{\prime} \backslash p^{-1}(N)$. It is easy to extend this contact structure over $M^{\prime}$ so that $p^{-1}(\Gamma)$ is a transverse link and the contact structure is the pull back of $\xi$ on $M^{\prime} \backslash p^{-1}(\Gamma)$. Call this contact structure $\xi^{\prime}$.

Lemma 6.5. Suppose $(M, \xi)$ is supported by a hyper-elliptic open book $(\Sigma, \phi)$. Then $M$ is a 2-fold branch cover over $S^{3}$ and $\xi$ is the pull-back of the standard tight contact structure on $S^{3}$.

Proof. We consider the case when $\Sigma$ has one boundary component, leaving the other, analogous case to the reader. Recall $M$ is the union of the mapping torus $T_{\phi}$ of $\phi$ and a solid torus $N$. The mapping cylinder is obtained from $\Sigma \times[0,1]$ by identifying $\Sigma \times\{0\}$ and $\Sigma \times\{1\}$ via $\phi$. The hyper-elliptic involution $h_{1}$ of $\Sigma$ induces a branch covering of $\Sigma \times[0,1]$ over $D^{2} \times[0,1]$, and since $\phi$ commutes with $h_{1}$ we obtain a branch covering of $T_{\phi}$ over $D^{2} \times S^{1}$. Note that this is a regular 2 -fold covering of $\partial T_{\phi}$ over $\partial D^{2} \times S^{1}$. The solid torus $N$ double covers the solid torus $S^{1} \times D^{2}$. Gluing $D^{2} \times S^{1}$ and $S^{1} \times D^{2}$ together (in the obvious way that preserves the product structure on the boundary) yields $S^{3}$, and the coverings maps on $T_{\phi}$ and $N$ fit together to give a branch covering of $M$ over $S^{3}$. It is also easy to see that the standard open book with disk pages for $S^{3}$ pulls back under this covering map to the open book for $M$. Thus $\xi$ is the pull-back of the standard contact structure on $S^{3}$.

Lemma 6.6. Let $(M, \xi)$ be a contact 3-manifold and $\Gamma$ a transverse link in it. Let $\left(M^{\prime}, \xi^{\prime}\right)$ be the 2 -fold cover of $(M, \xi)$ over $\Gamma$. Then

$$
e\left(\xi^{\prime}\right)=p^{*}(e(\xi)-u)
$$

where $u$ is the Poincaré dual of the homology class $[\Gamma]$.

Proof. Let $s: M \rightarrow \xi$ be a section of $\xi$ transverse to the zero section. Let $Z=$ $s^{-1}(0)$. The Poincaré dual of $e(\xi)$ is $[Z]$. Let $N$ be a neighborhood of $\Gamma$ as above. We can homotope $s$ so that $Z$ is disjoint from $N$. On $p^{-1}(M \backslash N)$, s will give a section $s^{\prime}$ of $\xi^{\prime}$ that is transverse to the zero section and $\left(s^{\prime}\right)^{-1}(0)=p^{-1}(Z)$. We now extend this section over $p^{-1}(N)$. To this end, note that each component of $N$ is $S^{1} \times D^{2}$ and we may homotope $\xi$ in $N$ so that it is tangent to the $D^{2}$ factor. (We have left the world of contact structures, but we are only trying to compute the Euler class of the plane field $\xi^{\prime}$, which can be done using any plane field homotopic to it.) For each point $p \in \Gamma$ the vector $s(p) \in \xi$ is transverse to $\Gamma$. If $N$ is taken to be a sufficiently small neighborhood of $\Gamma$, we may assume that $s(p)$ is the constant vector field on each $\{p t\} \times D^{2}$. Consider the branch map $D^{2} \rightarrow D^{2}: z \rightarrow z^{2}$. This map pulls back the constant vector field along $\partial D^{2}$ to a nonzero vector field near $\partial D^{2}$ with winding -1 . Thus it may be extended over $D^{2}$ to a vector field with one transverse zero. Hence the section $s$ on $\partial N$ induces a section of $\xi^{\prime}$ on $\partial p^{-1}(N)$ that can be extended over $p^{-1}(N)$ so that it is zero along $p^{-1}(\Gamma)$. We now have a section $s^{\prime}$ of $\xi^{\prime}$ defined on all of $M^{\prime}$ that is transverse to the zero section on $\left(s^{\prime}\right)^{-1}(0)=p^{-1}(Z)-p^{-1}(\Gamma)$. Taking Poincaré duals we obtain the desired formula. 
Remark 6.7. One may similarly derive a formula for more general branched covers. In particular, the formula for other cyclic covers and simple covers is a direct generalization of the one in Lemma 6.6.

Proof of Theorem 6.3. If $(M, \xi)$ is a contact manifold given by the hyper-elliptic open book $(\Sigma, \phi)$, then there is a 2 -fold branched covering $p: M \rightarrow S^{3}$ along $\Gamma \subset S^{3}$ such that $\xi=p^{*}\left(\xi_{s t d}\right)$. There is a surface $S$ in $S^{3}$ such that $\partial S=\Gamma$. The 2-fold branched cover $M$ is formed by gluing two copies of $S^{3} \backslash S$ together. Thus the branch set $\Gamma$ in $M$ is the boundary of $S \subset M$ and hence null-homologous. Lemma 6.6 now implies that $e(\xi)=p^{*} e\left(\xi_{s t d}\right)=p^{*} 0=0$.

\section{The NORM OF A CONTACT STRUCTURE}

In this section we discuss another invariant of contact structures derived from open book decompositions. Motivated by the Thurston norm we define the norm of a contact structure.

Definition 7.1. The norm of a contact structure $\xi$ on a 3 -manifold $M$ is the negative of the minimal Euler characteristic of a page of an open book supporting $\xi$,

$$
\mathrm{n}(\xi)=\min \{-\chi(\Sigma) \mid(\Sigma, \phi) \text { an open book decomposition supporting } \xi\} .
$$

For a given surface any pair of "genus", "number of boundary components" and "Euler characteristic" determine the third. However, it is not clear if the support genus and binding number of a contact structure determine the norm. We have the following simple observations.

Lemma 7.2. For any contact structure $\xi$ we have $\mathrm{n}(\xi) \leq-1$ with equality if and only if $\xi$ is the standard tight contact structure on $S^{3}$. Moreover, we have

$$
\min \{2 \operatorname{sg}(\xi)+\operatorname{bn}(\xi)-2,2 \operatorname{sg}(\xi)+1\} \leq \mathrm{n}(\xi) \leq 2 \operatorname{sg}(\xi)+\operatorname{bn}(\xi)-2 .
$$

Thus for contact structures with $\mathrm{bn}(\xi) \leq 3$ we know $\mathrm{n}(\xi)=2 \mathrm{sg}(\xi)+\mathrm{bn}(\xi)-2$.

Proof. The only nontrivial statement is the lower bound. If the minimum in the definition of $\mathrm{n}(\xi)$ is achieved with an open book with genus $g>\operatorname{sg}(\xi)$ and $m$ boundary components, then $\mathrm{n}(\xi)=2 g+m-2$. The smallest possible value for the right hand side is $2 \operatorname{sg}(\xi)+1$.

Corollary 7.3. For any contact structure $\xi$ in $S^{3}$ we have $n(\xi) \leq 4$.

Proof. For any contact structure $\xi$ in $S^{3}$ we have $\operatorname{sg}(\xi)=0$. The corollary follows by combining Lemma 5.5 with Lemma 7.2 .

Similarly the next result follows from Theorem 5.3 and Lemma 7.2.

Corollary 7.4. The norm of an overtwisted contact structure $\xi$ on a 3-manifold is bounded in terms of the Euler class of $\xi$. In particular, the infinitely many overtwisted contact structures on a manifold with Euler class zero (or torsional) take on finitely many norms. 


\section{Questions}

From [E1] and [OSS] we know the support genus has geometric meaning in that if $\operatorname{sg}(\xi)=0$ this forces any filling of $\xi$ to have certain properties and the HeegaardFloer invariant to have certain properties.

Question 8.1. Is there geometric content to the binding number and the norm of a contact structure?

It would be reasonable to suspect that the binding number has something to do with the homotopy class of the underlying plane field for the contact structure, but Theorem 5.3 shows that is not the case or at least that the binding number is insensitive to the homotopy class on the three skeleton. We ask

Question 8.2. On a manifold $M$ is there a bound on the binding number of any overtwisted contact structure (that does not depend on the Euler class, but only on $M)$ ?

One could also ask if the binding number of tight contact structures is bounded. For a specific question consider

Question 8.3. Let $\xi_{n}$ be the contact structure on $T^{3}$ with Giroux torsion $n$. Is $\operatorname{bn}\left(\xi_{n}\right)$ bounded independently of $n$ ?

Based on open book decompositions constructed by Van Horn [Vh] one might conjecture: $\operatorname{bn}\left(\xi_{n}\right)=3 n$.

Though there are countless other questions one could ask we end with

Question 8.4. Is the norm of a contact structure determined by the support genus and binding number?

\section{REFERENCES}

DGS. F. Ding, H. Geiges, and A. I. Stipsicz, Surgery diagrams for contact 3-manifolds, Turkish J. Math, 28 (2004) no. 1, 41-74. MR2056760 (2005c:57028)

E. Y. Eliashberg, Filling by holomorphic discs and its applications, Geometry of lowdimensional manifolds, 2 (Durham, 1989), London Math. Soc. Lecture Note Ser., 151 (1990), 45-67. MR1171908 (93g:53060)

E1. J. B. Etnyre, Planar open book decompositions and contact structures, IMRN 79 (2004), 4255-4267. MR2126827 (2005k:57049)

E2. J. B. Etnyre, Lectures on open book decompositions and contact structures, Lecture notes from the Clay Mathematics Institute Summer School on Floer Homology, Gauge Theory, and Low Dimensional Topology at the Alfréd Rényi Institute; Clay Math. Proc., 5, AMS, 2006. MR2249250 (2007g:57042)

EF. J. B. Etnyre and T. Fuller, Realizing 4-manifolds as achiral Lefschetz fibrations, Int. Math. Res. Not. 2006, Art. ID 70272. MR2219214 (2007d:57040)

EO. J. B. Etnyre and B. Ozbagci, Open books and plumbings, Int. Math. Res. Not. 2006, Art. ID 72710. MR2272094

Ge. H. Geiges, Constructions of contact manifolds, Math. Proc. Cambridge Philos. Soc. 121 (1997), no. 3, 455-464. MR1434654 (98f:53027)

Gi. E. Giroux, Géometrie de contact: de la dimension trois vers les dimensions supérieures, Proceedings of the International Congress of Mathematicians (Beijing 2002), Vol. II, 405414. MR1957051 (2004c:53144)

G. R. Gompf, Handlebody construction of Stein surfaces, Ann. of Math. (2), 148 (1998) no. 2, 619-693. MR1668563 (2000a:57070)

GS. R. Gompf and A. Stipsicz, 4-manifolds and Kirby calculus, Grad. Stud. Math., Vol. 20, AMS, 1999. MR1707327 (2000h:57038) 
HN. U. Hirsch and W.D. Neumann, On cyclic branched coverings of spheres, Math. Ann. 215 (1975), 289-291. MR0375321 (51:11516)

HKM. K. Honda, W. Kazez and G. Matic, Right-veering diffeomorphisms of compact surfaces with boundary I, preprint 2005.

NR. W.D. Neumann and L. Rudolph, Difference index of vector fields and the enhanced Milnor number, Topology 29 (1990) no. 1, 83-100. MR1046626 (91j:57010)

OS. B. Ozbagci and A. Stipsicz, Surgery on contact 3-manifolds and Stein surfaces, Bolyai Soc. Math. Stud., Vol. 13, Springer, 2004. MR2114165 (2005k:53171)

OSS. P. Ozsvath, A. Stipsicz and Z. Szabo, Planar open books and Floer homology, Int. Math. Res. Not. 2005, no. 54, 3385-3401. MR2200085 (2006j:57056)

Ro. D. Rolfsen, Knots and links, Publish or Perish, 1976. MR0515288 (58:24236)

S. S. Schönenberger, Planar open books and symplectic fillings, Ph.D. Dissertation, the University of Pennsylvania, 2005.

TW. W. Thurston and H. Winkelnkemper, On the existence of contact forms, Proc. Amer. Math. Soc. 52 (1975), 345-347. MR0375366 (51:11561)

Vh. J. Van Horn, Ph.D. Dissertation, the University of Texas at Austin, in preparation.

School of Mathematics, Georgia Institute of Technology, Atlanta, Georgia 30332

E-mail address: etnyre@math.gatech.edu

Department of Mathematics, KoÇ University, Istanbul, Turkey

E-mail address: bozbagci@ku.edu.tr 